\title{
E-Health and the Change of the Health Paradigm
}

\author{
David Naranjo Hernandez* \\ Biomedical Engineering Group of the University of Seville, Spain
}

*Corresponding author: David Naranjo Hernandez, Biomedical Engineering Group of the University of Seville, Spain.

Received Date: August 08, 2018

Published Date: August 27, 2018

\section{Editorial}

The demographic changes associated with the aging of the population are leading to a meaningful change in the social and economic structure of society. In 2030, it is expected that one third of European citizens will be over 65 years old and $40 \%$ of them will not be able to achieve a dignified quality of life independently. Living longer is not always synonymous with health and quality of life. From a certain age, health disorders are becoming more common chronic diseases, cardiovascular problems, dementia, etc. On the other hand, global demographic trends show a clear increase in the prevalence of chronic diseases.

The consequence of this growth, both in chronic diseases and in the number of elderly people, is an increase in the demand for healthcare, while financial and human resources are decreasing. The cost of health systems has risen to unsustainable levels, a circumstance that is even more accentuated by the effects of the economic crisis. While this problem is evident in developed countries, it is also becoming a major problem in developing countries. Thus, important public health challenges are posed, since most health systems have exceeded the available resources and do not have the capacity or framework to meet the growing demands of technology and healthcare services of an aging population that suffers from a higher incidence in chronic diseases. The situation is problematic and effective steps for a more cost-effective provision of health services are imperative.

Advances in the field of Information and Communication Technologies ICT have opened enormous opportunities for the improvement of citizens' health and well-being. e-Health is the term used to define the application of ICT in health care, as tools for prevention, diagnosis, treatment, monitoring and management of the health of the user or patient. E-Health technologies enable the development of applications that favour the adoption and maintenance of healthy lifestyles, the early diagnosis of health problems and the promotion of patient self-care. Its objective is to alleviate the overload of health systems and facilitate better care through patient follow-up, better diagnosis and intervention. This ability to assist the patients in the comfort of their own home can improve their quality of life, with the added benefit that patient care can cost less than $50 \%$, becoming the most appropriate option in many cases.

The new e-Health systems will represent a paradigm shift from the current model, which is centered around highly specialized experts focused on the care of acute patients, towards systems distributed around patients, which penetrate their daily lives and allow them to take an active part in the conservation and improvement of their health through self-care in the home. The adoption of preventive measures through the monitoring of the patient's health status and a better diagnosis and treatment will have a positive impact on a more efficient, more cost-effective and sustainable healthcare. This implies a paradigm shift towards a more personalized and proactive approach, in which patients participate actively in the decision-making process from the previous stages of the development of their illnesses. 